\section{G242 IMPROVED ANTENATAL DETECTION OF CRITICAL CONGENITAL HEART DISEASE RESULTS IN IMPROVED OUTCOME}

${ }^{1} \mathrm{~K}$ Khor, ${ }^{2,3} \mathrm{E}$ Parry, ${ }^{1} \mathrm{AK}$ Finucane, ${ }^{3} \mathrm{M}$ Harpham, ${ }^{1,3} \mathrm{TL}$ Gentles. ${ }^{1}$ Greenlane Paediatric and Congenital Cardiac Service, Starship Children's Hospital, Auckland, New Zealand; ${ }^{2}$ Department of Obstetrics and Gynaecology, National Women's Hospital, Auckland, New Zealand; ${ }^{3}$ New Zealand Maternal Fetal Medicine Network, National Women's Hospital, Auckland, New Zealand

\subsection{6/archdischild-2018-rcpch.235}

Introduction Isolated reports from selected populations suggest early diagnosis of critical congenital heart disease (cCHD) may result in improved outcome, especially in conditions where the surgical outcome is excellent; however, there are few population-based data to support this hypothesis. Furthermore, interpretation is frequently complicated by failure to account for noncardiac comorbidity that may drive outcome.

Methods We undertook a population-based review of newborns with cCHD born between 2006 and 2014. Cases were acquired from the National Fetal Cardiology and Cardiac Surgical databases and from the statutory reporting body for perinatal deaths. All diagnoses from 20 weeks gestation and diagnoses made at post-mortem were included. The timing of diagnosis, survival to cardiac surgery and 30 day mortality were reviewed in all livebirths where there was potential for a two-ventricle circulation Those with syndromes. additional major noncardiac anomalies were excluded from the analysis as were those born prematurely (i.e. $<35$ weeks gestation).

Results Of the 436 infants born with cCHD and a potential 2 ventricle circulation, 371 did not have a syndrome or a major non-cardiac abnormality.

The rate of antenatal diagnosis increased during the study period while the rate of postnatal diagnosis before and after hospital discharge declined $(\mathrm{p}=0.006)$.

The 30 day mortality rate declined from $7.0 \%$ to $0.9 \%$ during the study period $(p=0.049)$ and was largely confined to those who died prior to cardiac surgery.

Termination of pregnancy was uncommon and did not vary over the study period.

Conclusions There has been a significant increase in the rate of antenatal diagnosis in infants with a potential two ventricle circulation. This has been an associated decrease in 30 day mortality.

It is likely there are a number of factors responsible for these findings including earlier diagnosis allowing delivery at the cardiac surgical centre, and enhanced intensive care treatment before surgery.

\section{G243 A MORE EFFICIENT REGIONAL CARDIAC NETWORK: YES, WECCAN!}

P Mikrou, A Chikermane, M Chaudhari. Paediatric Cardiology Department, Birmingham Children's Hospital, Birmingham, UK

\subsection{6/archdischild-2018-rcpch.236}

Background and aims Outpatient paediatric cardiology services in the West Midlands Children's Cardiac Network (WeCCaN) consist of cardiology clinics in the tertiary centre and clinics in District General Hospitals (DGHs) run by either Consultant Paediatric Cardiologists (joint cardiology clinics) or by Paediatricians with Expertise in Cardiology (PECs). We aimed to assess the communication between tertiary cardiology, local Paediatricians and PECs and establish whether the cardiac network runs efficiently.

Methods We collected data from all patients seen in tertiary cardiology clinics over a period of one month (November 2016).

Results In total 1023 patients were seen between 01/11/2016 and 30/11/2016 in 108 Cardiology clinics and 12 specialist cardiac clinics. Age range was 3 weeks-19 years and M:F ratio was 1.3:1. Diagnosis was divided in: simple structural defects (35\%), complex structural defects (28\%), non-structural heart disease (21\%) and acquired/inherited disease (16\%). 195 patients were new (12\%) and 828 were follow-up (81\%). There was no cardiac pathology identified in $66 \%$ of new referrals. 369 patients $(36 \%)$ had underlying non-cardiac comorbidities.

Communication to PECs took place in $26 \%$ of new referrals and $34 \%$ of follow-up patients (postcode in a region with PEC service). Letters to General/Community Paediatrician were sent in $45 \%$ of new referrals and $53 \%$ of follow-up patients. Looking into disease severity and reasons for referral/followup, we concluded that $77 \%$ of new and $30 \%$ of follow-up patients could have seen a PEC instead of a Consultant Paediatric Cardiologist.

Conclusions

- Communication between tertiary Cardiology and PECs is suboptimal: We are currently working towards an information-sharing network, by providing access to our cardiac database for all West Midlands PECs.

- Communication with Paediatricians is suboptimal: We wish to approach Community Paediatricians and create a working relationship to ensure that all patients with complex heart disease undergo neurodevelopmental surveillance.

- $2 / 3$ rd of new patients and $1 / 3$ rd of follow-ups could have seen a PEC.

- 1 in 6 patients are under cardiac surveillance for acquired/ inherited disease: Re-designing cardiac services to include PEC and/or combined nurse practitioner/physiologist-led clinics in the tertiary centre is being discussed to improve efficiency and enhance workforce development.

\section{G244 HOW GOOD ARE PAEDIATRIC DOCTORS IN INTERPRETING ECGS}

L Prasad, R Talekar. General Paediatrics, Sheffield Children's Hospital NHS Trust, Sheffield, UK

10.1136/archdischild-2018-rcpch.237

Aim

- Conduct a survey among Yorkshire paediatric doctors and identify deficiencies in paediatric ECG interpretation skills and knowledge.

- Assess the need for a paediatric ECG e-module.

Methods An online questionnaire was sent out to all Yorkshire Paediatric doctors. The questionnaire dealt with topics ranging from lead placements to common paediatric abnormalities in seperate sections. In each subsection, subjective questions relating to confidence in interpretation were followed by objective questions. Participants were also asked about the usefulness of a problem based ECG e-module and were asked to provide suggestions for topics to be included.

Results Total numbers: 


\begin{tabular}{lllll}
\multicolumn{4}{l}{ Abstract G244 } & Table 1 \\
\hline ST1-3 & ST4-6 & ST6+ & Consultant & Total \\
\hline 41 & 47 & 23 & 18 & 129 \\
$(31.78 \%)$ & $(36.4 \%)$ & $(17.82 \%)$ & $(13.95 \%)$ & \\
\hline
\end{tabular}

None of the responders answered all the questions correctly. There were wide discrepancies between objective and subjective knowledge of several aspects of ECGs.

Subjective interpretation of ECG axis scored the lowest with none of the level 1 doctors stating that they were confident; objective scores were better for this group at $21.8 \%$. The highest scores in the objective section were for calculating rates (100\% in the level 2 group).

Only $69 \%$ of the level 1 doctors said they were confident in lead placement against $87 \%$ of the consultants. Level 1 doctors scored only $51 \%$ in objective questions dealing with lead placement.

Confidence in interpretation of common ECG abnormalities was low with level 1 doctors at 5\% and consultants at 36\%. Objective scores scored better. ECGs on myocarditis, SVT and heart block scored better over pre-excitation, axis deviation and hypertrophy.

ECG showing Q waves were correctly identified by only 3 responders. Only $34 \%$ of the responders identified QTC interval as abnormal.

Responses were not always consistent with level of training and hade wide variations.

93\% of doctors responded favourably to an e-module for paediatric ECGs.Frequent requests for topics included age related changes, neonatal ECGs, tachyarrythmias, axis and QT interval interpretation.

Conclusions This survey revealed gaps and variations in ECG interpretation skills among paediatric doctors. Majority felt that an online problem based e-module on paediatric ECG interpretation would help in improving knowledge and confidence. This will be developed and uploaded to the e-learning for health care(ww.e-lfh.org.uk)website.

\section{G245 RISK FACTORS FOR ABNORMAL DEVELOPMENT AND COGNITIVE FUNCTION IN CHILDREN WITH CONGENITAL HEART DISEASE AND IMPLICATIONS FOR SERVICE PROVISION IN THE UK}

${ }^{1} \mathrm{~V}$ Banks, ${ }^{1} \mathrm{~A}$ Hoskote, ${ }^{1} \mathrm{~S}$ Kakat, ${ }^{2} \mathrm{D}$ Rideout, ${ }^{2} \mathrm{M}$ Lakhanpaul, ${ }^{2} \mathrm{C}$ Pagel, ${ }^{3} \mathrm{R}$ Franklin,
${ }^{4} \mathrm{~T}$ Witter. ${ }^{1}$ Cardiorespiratory Unit, Great Ormond Street Hospital, London, UK; ${ }^{2}$ University
College London, London, UK; ${ }^{3}$ Royal Brompton Hospital, London, UK; ${ }^{4}$ Evelina Childrens
Hospital, London, UK

10.1136/archdischild-2018-rcpch.238

Background Within the NHS context there is currently no specific additional neurodevelopmental follow up for children with congenital heart disease (CHD). Cardiologists are not trained in neurodevelopment and face many competing pressures, which may result in delayed detection of problems. We aimed to assess whether there is appropriate service provision for children with CHD and abnormal developmental outcome in the UK, and identify risk factors that could trigger early referral and more timely intervention.

Methods Usingvalidated scales of developmental/cognitive function with children less than 17 years of age with CHD treated at one of 3 centres in London, we assessed whether those with abnormal scores (defined as $>2$ standard deviations below the mean) were under follow up by appropriate developmental services. Logistic regression was used to identify potential socio-economic and clinical risk factors for adverse developmental and cognitive outcomes.

Results Being under follow up by developmental services was strongly associated $(\mathrm{p}<0.001)$ with abnormal developmental/ cognitive score but over $50 \%$ of patients with abnormal scores were not under any appropriate services. Risk factors associated with abnormal development/cognitive scores differed across age groups. Greater severity of CHD was associated with adverse gross motor development. Socio-economic factors were linked to adverse neurodevelopmental outcomes in older children.

Conclusion We identified a large number of patients with abnormal development not currently under appropriate care services, highlighting a potential failure in the care pathway for these children. A number of risk factors for adverse neurodevelopment were identified which could provide useful insight for clinicians to ensure high risk patients are appropriately referred, ensuring more timely assessment and intervention to enhance future development and quality of life for these children.

\section{G246(P) CLOSING THE DUCTUS ARTERIOSUS IN PRETERM INFANTS. A REVIEW OF PRESENT TREATMENT STRATEGIES AND DEVELOPING A DISEASE STAGING PROTOCOL}

H Raychaudhuri, A Bahadur, M Alam, S Nayak, I Ali. Neonatology, Newham Hospital, Barts and London NHS Trust, London, UK

10.1136/archdischild-2018-rcpch.239

Background The ductus arteriosus (DA) is an important structure in foetal life. Closure of the DA is an essential part of postnatal adaptation. Closure of the DA is initiated by an increase in oxygen and changes in pulmonary and systemic blood pressure. In preterm infants, failure of DA closure after birth can be associated with an increased incidence of chronic lung disease (CLD), intraventricular haemorrhage (IVH) and necrotizing enterocolitis (NEC). Prostaglandin inhibition using indomethacin or ibuprofen is the standard strategy to close the DA. Surgical closure and interventional device closure of the DA are an alternative option. Appropriate timing for closing the duct still remains a debatable topic. Various staging methods have been proposed based on Echocardiographic and clinical parameters to help clinicians make a decision. This study aims at assessing the effect of closing a DA on the overall morbidity and mortality in preterm infants and further interrogating available evidence on the best practice and optimum time for closing the DA. We designed a PDA staging protocol based on available evidence which will help clinicians decide on closing the DA.

Methods We conducted a review of literature and results from 10 different papers were assessed and analysed for this study.

Conclusion Closure of the DA in the first few weeks of life may provide short-term benefits. The long-term effects of untreated PDA in extremely premature infants remain unclear. Significant changes in management have evolved in the recent years including early surgical ligation and transcutaneous device closure but the evidence to support these changes are 